\title{
The variation of the maximal function of a radial function
}

\author{
Hannes Luiro
}

\begin{abstract}
It is shown for the non-centered Hardy-Littlewood maximal operator $M$ that $\|D M f\|_{1} \leq C_{n}\|D f\|_{1}$ for all radial functions in $W^{1,1}\left(\mathbb{R}^{n}\right)$.
\end{abstract}

\section{Introduction}

The non-centered Hardy-Littlewood maximal operator $M$ is defined by setting for $f \in L_{\text {loc }}^{1}\left(\mathbb{R}^{n}\right)$ that

$$
M f(x)=\sup _{B(z, r) \ni x} \frac{1}{|B(z, r)|} \int_{B(z, r)}|f(y)| d y=: \sup _{B(z, r) \ni x} f_{B(z, r)}|f(y)| d y
$$

for every $x \in \mathbb{R}^{n}$. The centered version of $M$, denoted by $M_{c}$, is defined by taking the supremum over all balls centered at $x$. The classical theorem of Hardy, Littlewood and Wiener asserts that $M$ (and $M_{c}$ ) is bounded on $L^{p}\left(\mathbb{R}^{n}\right)$ for $1<p \leq \infty$. This result is one of the cornerstones of the harmonic analysis. While the absolute size of a maximal function is usually the principal interest, the applications in Sobolev-spaces and in the potential theory have motivated the active research of the regularity properties of maximal functions. The first observation was made by Kinnunen who verified [Ki] that $M_{c}$ is bounded in Sobolev-space $W^{1, p}\left(\mathbb{R}^{n}\right)$ if $1<p \leq \infty$, and inequality

$$
\left|D M_{c} f(x)\right| \leq M_{c}(|D f|)(x)
$$

holds for all $x \in \mathbb{R}^{n}$. The proof is relatively simple and inequality (1.2) (and the boundedness) holds also for $M$ and many other variants.

The author was supported by the Academy of Finland, project no. 292797.

2010 Mathematics Subject Classification: 42B25, 46E35, 26A45. 
The most challenging open problem in this field is so called ' $W^{1,1}$-problem': Does it hold for all $f \in W^{1,1}\left(\mathbb{R}^{n}\right)$, that $D M f \in L^{1}\left(\mathbb{R}^{n}\right)$ and

$$
\|D M f\|_{1} \leq C_{n}\|D f\|_{1} ?
$$

This problem has been discussed (and studied) for example in [AlPe], [CaHu], [CaMa], $[\mathrm{HO}],[\mathrm{HM}],[\mathrm{Ku}]$ and $[\mathrm{Ta}]$. The fundamental obstacle is that $M$ is not bounded in $L^{1}$ and therefore inequality (1.2) is not enough to solve the problem. In the case $n=1$ the answer is known to be positive, as was proved by Tanaka [Ta]. For $M_{c}$ the problem turns out to be very complicated also when $n=1$; however, Kurka $[\mathrm{Ku}]$ managed to show that the answer is positive also in this case.

The goal of this paper is to develop technology for $W^{1,1}$-problem in higher dimensions, where the problem is still completely open. The known proofs in the one-dimensional case are strongly based on the simplicity of the topology: the crucial trick (in the non-centered case) is that $M f$ does not have a strong local maximum (Definition 3.7) outside the set $\{M f(x)=f(x)\}$. This fact is a strong tool when $n=1$ but is far from sufficient for higher dimensions.

The formula for the derivative of the maximal function (see Lemma 2.2 or $[\mathrm{L}]$ ) has an important role in the paper. It says that if $M f(x)=f_{B}|f|,|f(x)|<M f(x)<$ $\infty$, and $M f$ is differentiable at $x$, then

$$
D M f(x)=f_{B} D f(y) d y .
$$

From this formula one can see immediately the validity of the estimate (1.2) for $M$; however, since $B$ is exactly the ball which gives the maximal average (for $|f|$ ), it is expected that one can derive from (1.3) much more sophisticated estimates than (1.2). In Section 2 (Lemma 2.2), we perform basic analysis related to this issue. The key observation we make is that if $B$ is as above, then

$$
\int_{B} D f(y) \cdot(y-x) d y=0 .
$$

In the backround of this equality stands a more general principle, concerning other maximal operators as well: if the value of the maximal function is attained to ball (or other permissible object) $B$, then the weighted integral of $|D f|$ over $B$ is zero for a set of weights depending on the maximal operator. We believe that the utilization of this principle is a key for a possible solution of $W^{1,1}$-problem.

As the main result of this paper, we employ equality (1.4) to show that in the case of radial functions the answer to $W^{1,1}$-problem is positive (Theorem 3.12). Even in this case, the problem is evidently non-trivial and truly differs from the onedimensional case. To become convinced about this, consider the important special 
case where $f$ is radially decreasing $(f(x)=g(|x|)$, where $g:[0, \infty) \rightarrow \mathbb{R}$ is decreasing). In this case, $M f$ is radially decreasing as well and $M f(0)=f(0)$. If $n=1$, these facts immediately imply that $\|D M f\|_{1}=\|D f\|_{1}$, but if $n \geq 2$ this is definitely not the case: the additional estimates are necessary. This type of estimate for radially decreasing functions can be derived from (1.3) and (1.4), saying that

$$
|D M f(x)| \leq \frac{C_{n}}{|x|} f_{B(0,|x|)}|D f(y)||y| d y .
$$

By using this inequality, the positive answer to $W^{1,1}$-problem for radially decreasing functions follows straightforwardly by Fubini Theorem (Theorem 3.4).

For general radial functions, inequality (1.5) turns out to hold only if the maximal average is achieved in a ball with radius comparable to $|x|$. To overcome this problem, we study the auxiliary maximal function $M^{I}$, defined for $f \in L_{\mathrm{loc}}^{1}\left(\mathbb{R}^{n}\right)$ by

$$
M^{I} f(x)=\sup _{x \in B(z, r), r \leq|z| / 4} f_{B(z, r)}|f(y)| d y,
$$

and prove (Lemma 3.5) that for all radial $f \in W^{1,1}\left(\mathbb{R}^{n}\right)$ it holds that

$$
\left\|D M^{I} f\right\|_{1} \leq C_{n}\|D f\|_{1}
$$

The proof of this auxiliary result resembles the proof of $W^{1,1}$-problem (for $M$ ) in the case $n=1$. Recall again that in the case $n=1$ the key is that $M f$ does not have a strong local maximum in $\{M f(x)>|f(x)|\}$. As a multidimensional counterpart for radial functions, we show that $M^{I} f$ does not have a strong local maximum in $\left\{M^{I} f(x)>|f(x)|\right\}$ and for every $k \in \mathbb{Z}$ it holds that

$$
\int_{\left\{2^{k} \leq|y| \leq 2^{k+1}\right\}}\left|D M^{I} f(y)\right| d y \leq C_{n} \int_{\left\{2^{k-1} \leq|y| \leq 2^{k+2}\right\}}|D| f|(y)| d y .
$$

Estimate (1.6) can be easily derived from this fact. The main result follows by combining (1.6) and exploiting the estimate (1.5) in $\left\{M f(x)>M^{I} f(x)\right\}$.

\section{Question}

The analysis presented in this paper raises the interest towards the study of the integrability properties of some conditional maximal operators. As an example, (1.3) and (1.4) yield that $|D M f(x)| \leq \widetilde{M}(D|f|)(x)$, where $\widetilde{M}$ is defined for all locally integrable gradient fields $F: \mathbb{R}^{n} \rightarrow \mathbb{R}^{n}$ by

$$
\widetilde{M} F(x)=\sup \left\{\left|f_{B(z, r)} F\right|: x \in B(z, r), \int_{B(z, r)} F(y) \cdot(y-x) d y=0\right\} .
$$


It is clear that $\widetilde{M} F$ is bounded by $M(|F|)$, but does it hold that $\widetilde{M}$ has even better integrability properties than $M$ ? What about the boundedness in the Hardy-space $H^{1}$ or even in $L^{1}$ ? Notice that the boundedness of $\widetilde{M}$ in $L^{1}$ would imply the solution to $W^{1,1}$-problem. This problem is almost completely open, even in the case $n=1$. Counterexamples would be highly interesting as well.

Acknowledgements. The author would like to thank Antti Vähäkangas for inspiring discussions and encouragement, and the anonymous referee for many valuable comments on the manuscript.

\section{Preliminaries and general results}

Let us introduce some notation. The boundary of the $n$-dimensional unit ball is denoted by $S^{n-1}$. The $s$-dimensional Hausdorff measure is denoted by $\mathcal{H}^{s}$. The volume of the $n$-dimensional unit ball is denoted by $\omega_{n}$ and the $\mathcal{H}^{n-1}$-measure of $S^{n-1}$ by $\sigma_{n}$. The weak derivative of $f$ (if exists) is denoted by $D f . D f(x)$ may also denote the classical derivative of $f$ at $x$, in the case it is known to exist. If $v \in S^{n-1}$, then

$$
D_{v} f(x):=\lim _{h \rightarrow 0} \frac{1}{h}(f(x+h v)-f(x)),
$$

in the case the limit exists.

Definition 2.1. For $f \in L_{\text {loc }}^{1}\left(\mathbb{R}^{n}\right)$ let

$$
\mathcal{B}_{x}:=\left\{B(z, r): x \in \bar{B}(z, r), r>0, f_{B}|f|=M f(x)\right\} .
$$

It is easy to see that if $f \in L^{1}\left(\mathbb{R}^{n}\right), x$ is a Lebesgue point for $f$, and $|f(x)|<$ $M f(x)<\infty$, then $\mathcal{B}_{x} \neq \varnothing$.

The following lemma is the main result of this section. We point out that below (6) is especially useful in the case of radial functions.

Lemma 2.2. If $f \in W^{1,1}\left(\mathbb{R}^{n}\right), M f(x)>|f(x)|$ and $M f$ is differentiable at $x$, then

(1) For all $v \in S^{n-1}$ and $B \in \mathcal{B}_{x}$, it holds that

$$
D M f(x)=f_{B} D|f|(y) d y \quad \text { and } \quad D_{v} M f(x)=f_{B} D_{v}|f|(y) d y .
$$

(2) If $x \in B$ for some $B \in \mathcal{B}_{x}$, then $\operatorname{DMf}(x)=0$.

(3) If $x \in \partial B, B=B(z, r) \in \mathcal{B}_{x}$ and $D M f(x) \neq 0$, then

$$
\frac{D M f(x)}{|D M f(x)|}=\frac{z-x}{|z-x|} \text {. }
$$


1. If $B \in \mathcal{B}_{x}$, then

$$
\int_{B} D|f|(y) \cdot(y-x) d y=0
$$

(4) If $x \in \partial B, B=B(z, r) \in \mathcal{B}_{x}$, then

$$
|D M f(x)|=\frac{1}{r} f_{B} D|f|(y) \cdot(z-y) d y .
$$

(5) If $B \in \mathcal{B}_{x}$, then

$$
D M f(x) \cdot \frac{x}{|x|}=\frac{1}{|x|} f_{B} D|f|(y) \cdot y d y .
$$

The proof of Lemma 2.2 is essentially based on the following auxiliary propositions.

Proposition 2.3. Suppose that $f \in W^{1,1}\left(\mathbb{R}^{n}\right), B$ is a ball, $h_{i} \in \mathbb{R}$ such that $h_{i} \rightarrow 0$ as $i \rightarrow \infty$, and $B_{i}=L_{i}(B)$, where $L_{i}$ are affine mappings and

$$
\lim _{i \rightarrow \infty} \frac{L_{i}(y)-y}{h_{i}}=g(y) .
$$

Then

$$
\lim _{i \rightarrow \infty} \frac{1}{h_{i}}\left(f_{B_{i}} f(y) d y-f_{B} f(y) d y\right)=f_{B} D f(y) \cdot g(y) d y .
$$

Proof. The proof is a simple calculation:

$$
\begin{aligned}
& \frac{1}{h_{i}}\left(f_{B_{i}} f(y) d y-f_{B} f(y) d y\right)=\frac{1}{h_{i}}\left(f_{L_{i}(B)} f(y) d y-f_{B} f(y) d y\right) \\
= & \frac{1}{h_{i}}\left(f_{B} f\left(L_{i}(y)\right)-f(y) d y\right)=f_{B} \frac{f\left(y+\left(L_{i}(y)-y\right)\right)-f(y)}{h_{i}} d y \\
\approx & f_{B} \frac{D f(y) \cdot\left(L_{i}(y)-y\right)}{h_{i}} d y \longrightarrow f_{B} D f(y) \cdot g(y) d y,
\end{aligned}
$$

if $i \rightarrow \infty$.

Lemma 2.4. Let $f \in W^{1,1}\left(\mathbb{R}^{n}\right), x \in \mathbb{R}^{n}, B \in \mathcal{B}_{x}, \delta>0$, and let $L_{h}, h \in[-\delta, \delta]$, be affine mappings such that $x \in L_{h}(\bar{B})$ and

$$
\lim _{h \rightarrow 0} \frac{L_{h}(y)-y}{h}=g(y) .
$$

Then

$$
\int_{B} D|f|(y) \cdot g(y) d y=0
$$


Proof. Let us denote $B_{h}:=L_{h}(B)$. By Proposition 2.3 it holds that

$$
f_{B} D|f|(y) \cdot g(y) d y=\lim _{h \rightarrow 0} \frac{1}{h}\left(f_{B_{h}}|f|(y)-f_{B}|f|(y)\right) .
$$

Since $B \in \mathcal{B}_{x}$ and $x \in \bar{B}_{h}$, the sign of the quantity inside the large parentheses is non-positive for all $h \in[-\delta, \delta]$; however, the sign of $1 / h$ depends on the sign of $h$. The conclusion is that the above equality is possible only if (2.11) is valid.

\section{Proof of Lemma 2.2}

(1) The claim is counterpart for the formula for $D M_{c} f$, which was first time proved in [L]. Suppose that $B=B(z, r) \in \mathcal{B}_{x}$ and let $B_{h}:=B(z+h v, r)$. Then it holds that

$$
\begin{aligned}
D_{v} M f(x) & =\lim _{h \rightarrow 0} \frac{1}{h}(M f(x+h v)-M f(x)) \\
& \geq \lim _{h \rightarrow 0} \frac{1}{h}\left(f_{B_{h}}|f(y)| d y-f_{B}|f(y)| d y\right) \\
& =\lim _{h \rightarrow 0} \frac{1}{h}\left(f_{B}|f(y+h v)|-|f(y)| d y\right)=f_{B} D_{v}|f|(y) d y .
\end{aligned}
$$

On the other hand, if $B_{h}:=B(z-h v, r)$, then

$$
\begin{aligned}
D_{v} M f(x) & =\lim _{h \rightarrow 0} \frac{1}{h}(M f(x)-M f(x-h v)) \\
& \leq \lim _{h \rightarrow 0} \frac{1}{h}\left(f_{B}|f(y)| d y-f_{B_{h}}|f(y)| d y\right) \\
& =\lim _{h \rightarrow 0} \frac{1}{h}\left(f_{B}|f(y)|-|f(y+h v)| d y\right)=f_{B} D_{v}|f|(y) d y .
\end{aligned}
$$

These inequalities imply the claim.

(2) If $B \in \mathcal{B}_{x}$ and $x \in B$, then $y \in B$ if $|y-x|$ is small enough, and thus $\operatorname{Mf}(y) \geq$ $M f(x)$.

(3) Let $B=B(z, r) \in \mathcal{B}_{x}, v \in S^{n-1}$ such that $v \cdot(z-x)=0$, and let us denote for all $h \in(0, \infty)$ that $x_{h}:=x+h v, r_{h}:=\left|z-x_{h}\right|$, and $B_{h}:=B\left(z, r_{h}\right)$. These definitions guarantee that $x_{h} \in \bar{B}_{h} \backslash B$ for all $h$, and $B \subset B_{h}$. Moreover, since $v \cdot(z-x)=0$, it is elementary fact that

$$
r_{h}=|z-x-h v| \leq|z-x|+\frac{h^{2}}{2 r} .
$$


Therefore, $r / r_{h} \geq 1-\left(\frac{h}{r}\right)^{2}$, and

$$
\begin{aligned}
M f\left(x_{h}\right) & \geq f_{B_{h}}|f(z)| d z \geq \frac{|B|}{\left|B_{h}\right|} f_{B}|f(z)| d z=\left(\frac{r}{r_{h}}\right)^{n} f_{B}|f(z)| d z \\
& \geq\left(1-\frac{h^{2}}{r^{2}}\right)^{n} M f(x) .
\end{aligned}
$$

This implies that $D_{v} M f(x) \geq 0$ for all $v \in S^{n-1}$ such that $v \cdot(z-x)=0$. Since we assumed that $M f$ is differentiable at $x$, it follows that

$$
D_{v} M f(x)=0 \quad \text { if } v \in S^{n-1}, v \cdot(z-x)=0 .
$$

In particular, it follows that $D M f(x)$ is parallel to $z-x$ or $x-z$. The final claim follows easily by the fact that $M f(x+h(z-x)) \geq M f(x)$ if $0 \leq h \leq 2$.

(4) Let $B \in \mathcal{B}_{x}$ and $L_{h}(y):=y+h(y-x), h \in \mathbb{R}$. Then it holds that $L_{h}$ is affine mapping, $L_{h}(x)=x$, and so $x \in L_{h}(B)=: B_{h}$, and $\left(L_{h}(y)-y\right) / h=y-x$ for all $h \in \mathbb{R}$. Therefore, Lemma 2.4 implies that

$$
\int_{B} D|f|(y) \cdot(y-x) d y=0 .
$$

(5) By combining (1), (3) and (4) the claim follows by

$$
\begin{aligned}
|D M f(x)| & =D M f(x) \cdot\left(\frac{z-x}{|z-x|}\right)=f_{B} D|f|(y) \cdot\left(\frac{z-x}{|z-x|}\right) d y \\
& =f_{B} D|f|(y) \cdot\left(\frac{z-y}{|z-x|}\right) d y .
\end{aligned}
$$

(6) The claim follows from (1) and (4).

\section{3. $W^{1,1}$-problem for radial functions}

\section{Radial functions and notation}

In what follows, we will interpret a radial function on $\mathbb{R}^{n}$ as a function on $(0, \infty)$ in a natural way. To be more precise, if $f \in W_{\text {loc }}^{1,1}\left(\mathbb{R}^{n}\right)$ is radial, it is well known fact that there exists continuous function $\tilde{f}:(0, \infty) \rightarrow \mathbb{R}$ such that $\tilde{f}$ is weakly differentiable,

$$
\int_{0}^{\infty}\left|\tilde{f}^{\prime}(t)\right| t^{n-1} d t<\infty,
$$

and (by a possible redefinition of $f$ in a set of measure zero) for all $t \in(0, \infty)$ it holds that $f(x)=\tilde{f}(t)$ and $D_{x /|x|} f(x)=\tilde{f}^{\prime}(t)$ if $|x|=t$. In what follows, we will simplify the 
notation and use $f$ to denote $\tilde{f}$ as well. To avoid the possibility of misunderstanding, we usually use variable $t$ and notation $f^{\prime}$ (instead of $D f$ ) when we are actually working with $\tilde{f}$. We also say that $f$ is radially decreasing if $f$ is radial and $f\left(t_{1}\right)<$ $f\left(t_{2}\right)$ if $t_{1}>t_{2}$. Notice also that if $f$ is radial then $M f$ is also radial.

We begin with establishing couple of auxiliary lemmas. The following auxiliary result is repeatedly utilized in the proof. The proof is well known, see for example [HKM, Theorem 1.20].

Lemma 3.1. Suppose that $\Omega \subset \mathbb{R}^{n}, f \in W^{1,1}(\Omega)$ is continuous, $g: \Omega \rightarrow \mathbb{R}$ is continuous and weakly differentiable in $E:=\{x \in \Omega: g(x)>f(x)\}$, and $\int_{E}|D g|<\infty$. Then $\max \{f, g\}$ is weakly differentiable in $\Omega$ and

$$
D(\max \{f, g\})=\chi_{E} D g+\chi_{\Omega \cap E^{c}} D f .
$$

Let us define an auxiliary maximal operator $M_{\lambda}$ for $\lambda>0$ by

$$
M_{\lambda} f(x)=\sup _{x \in B(z, r), \lambda \leq r} f_{B(z, r)}|f(y)| d y .
$$

Proposition 3.2. If $f \in L^{1}\left(\mathbb{R}^{n}\right)$, then $M_{\lambda}$ is Lipschitz.

Proof. The result is well known, but we give a proof for readers convenience. Suppose that $x, y \in \mathbb{R}^{n}$ such that $M_{\lambda} f(x)>M_{\lambda} f(y)$. Clearly there exists $r \geq \lambda$ and $x_{0} \in \mathbb{R}^{n}$ such that $x \in \bar{B}\left(x_{0}, r\right)$ and $M_{\lambda} f(x)=f_{B\left(x_{0}, r\right)}|f|$. The claim follows by

$$
\begin{aligned}
& M_{\lambda} f(x)-M_{\lambda} f(y) \leq f_{B\left(x_{0}, r\right)}|f(z)| d z-f_{B\left(x_{0}, r+|x-y|\right)}|f(z)| d z \\
\leq & \frac{1}{\omega_{n}}\left(\frac{1}{r^{n}}-\frac{1}{(r+|x-y|)^{n}}\right) \int_{B\left(x_{0}, r\right)}|f(z)| d z \leq C(n, \lambda)|x-y|\|f\|_{1} .
\end{aligned}
$$

The following result is especially related to the assumption ' $M f(x)$ is differentiable at $x^{\prime}$ in Lemma 2.2 .

Proposition 3.3. Suppose that $f \in W^{1,1}\left(\mathbb{R}^{n}\right)$ is radial and

$$
E:=\left\{x \in \mathbb{R}^{n} \backslash\{0\}: M f(x)>|f(x)|\right\} .
$$

Then $E$ is open, DMf exists in $E$ and $M f$ is differentiable almost everywhere in $E$.

Proof. The first claim ( $E$ is open) follows by the fact that $f$ is continuous outside the origin. The claims concerning the differentiability (weak and classical) follow if we can show that $M f$ is locally Lipschitz in $E$. But this follows rather easily from Proposition 3.2 and the fact that $f$ is continuous in $\mathbb{R}^{n} \backslash\{0\}$. 
The following result is a straightforward consequence of Lemma 2.2 and the above auxiliary results.

Theorem 3.4. If $f \in W^{1,1}\left(\mathbb{R}^{n}\right)$ is radially decreasing, then $D M f \in W^{1,1}\left(\mathbb{R}^{n}\right)$ and $\|D M f\|_{1} \leq C_{n}\|D f\|_{1}$.

Proof. Since $f$ is radially decreasing, it follows that $M f(x)>|f(x)|$ for all $x \neq 0$. Especially, this guarantees the existence of a weak derivative in $\mathbb{R}^{n} \backslash\{0\}$, and the classical differentiability almost everywhere (by the above auxiliary results).

If $B \in \mathcal{B}_{x}, x \neq 0$, it is easy to show (the proof is left to the reader) that $\bar{B} \subset$ $\bar{B}(0,|x|)$. It also follows that $0 \in \bar{B}$. To see this, observe (e.g.) that whenever $0 \notin \bar{B}$, $B \subset B(0,|x|)$, then $B$ is of type $B=B(c x,|c-1||x|)$, where $\frac{1}{2}<c<1$. By choosing

$$
L(y)=x+2(1-c)(y-x) \quad \text { and } \quad B^{*}:=B\left(\frac{1}{2} x, \frac{1}{2}|x|\right)
$$

it is easy to check that $L\left(B^{*}\right)=B$ and, especially, $|L(y)|>|y|$ for all $y \in B^{*}$. Therefore,

$$
f_{B}|f(z)| d z=f_{L\left(B^{*}\right)}|f(z)| d z=f_{B^{*}}|f(L(z))| d z<f_{B^{*}}|f(z)| d z
$$

This proves that $0 \in \bar{B}(x)$, whenever $B \in \mathcal{B}_{x}, x \neq 0$. Especially, we get by Lemma 2.2, (6) that

$$
|D M f(x)| \leq \frac{C_{n}}{|x|} f_{B(0,|x|)}|D f(y)||y| d y \quad \text { for a.e. } x .
$$

Then the claim follows by Fubini theorem:

$$
\begin{aligned}
& \int_{\mathbb{R}^{n}}\left(\frac{1}{|x|} f_{B(0,|x|)}|D f(y)||y| d y\right) d x \\
= & \int_{\mathbb{R}^{n}}|D f(y)||y|\left(\int_{\mathbb{R}^{n}} \frac{\chi_{B(0,|x|)}(y)}{\omega_{n}|x|^{n+1}} d x\right) d y \\
= & \int_{\mathbb{R}^{n}}|D f(y)||y|\left(\int_{\{x:|x| \geq|y|\}} \frac{1}{\omega_{n}|x|^{n+1}} d x\right) d y \\
= & \int_{\mathbb{R}^{n}}|D f(y)||y|\left(\int_{S^{n-1}} \int_{|y|}^{\infty} \frac{1}{\omega_{n} t^{n+1}} t^{n-1} d t d \mathcal{H}^{n-1}\right) d y \\
= & n \int_{\mathbb{R}^{n}}|D f(y)||y|\left(\int_{|y|}^{\infty} \frac{1}{t^{2}} d t\right) d y \\
= & n \int_{\mathbb{R}^{n}}|D f(y)| d y . \quad \square
\end{aligned}
$$


In the case of general radial functions, (1.5) is in general valid (and useful) only for those $x$ for which the radius of $B \in \mathcal{B}_{x}$ is comparable to $|x|$. As it was explained in the introduction, the main auxiliary tool in the case of general radial functions is the following result (recall the definition of $M^{I}$ in the introduction):

Lemma 3.5. If $f \in W^{1,1}\left(\mathbb{R}^{n}\right)$ is radial, then $M^{I} f \in W^{1,1}\left(\mathbb{R}^{n}\right)$ and $\left\|D M^{I} f\right\|_{1} \leq$ $C_{n}\|D f\|_{1}$.

Before the actual proof of this result, we prove several auxiliary results. The first of them is well known.

Proposition 3.6. Suppose that $E \subset \mathbb{R}$ is open. Then there exist disjoint intervals $\left(a_{i}, b_{i}\right)$ such that $E=\cup_{i=1}^{\infty}\left(a_{i}, b_{i}\right)$ and $a_{i}, b_{i} \in \partial E \cup\{-\infty, \infty\}$ for all $i \in \mathbb{N}$.

Definition 3.7. Let $f: \Omega \rightarrow \mathbb{R}$, where $\Omega \subset \mathbb{R}$ is open. We say that $x$ is a strong local maximum of $f$ in $(a, b) \subset \Omega,-\infty \leq a<b \leq \infty$, if there exist $a^{\prime}, b^{\prime} \in(a, b)$ such that $a^{\prime}<x<b^{\prime}, f(t) \leq f(x)$ if $t \in\left(a^{\prime}, b^{\prime}\right)$, and $\max \left\{f\left(a^{\prime}\right), f\left(b^{\prime}\right)\right\}<f(x)$.

Proposition 3.8. Suppose that $f:[a, b] \rightarrow \mathbb{R}$ is continuous and $c \in(a, b)$ such that $f(c)>\max \{f(a), f(b)\}$. Then $f$ has a strong local maximum on $(a, c)$.

Proof. It is easy to see that now any maximum point $c(f(c)=\max f)$, which is known to exist, is also a strong local maximum of $f$.

Proposition 3.9. Suppose that $f:[a, b] \rightarrow \mathbb{R}$ is continuous and does not have a strong local maximum on $(a, b)$. Then there exists $c \in[a, b]$ such that $f$ is nonincreasing on $[a, c]$ and non-decreasing on $[c, b]$.

Proof. Since $f$ is continuous, we can choose $c \in[a, b]$ such that $f(c)=\min f$. To show that $f$ is non-decreasing on $[c, b]$, let $c<y_{1}<y_{2}<b$ and assume, on the contrary, that $f\left(y_{2}\right)<f\left(y_{1}\right)$. This implies that $f\left(y_{1}\right)>\max \left\{f(c), f\left(y_{2}\right)\right\}$, and thus $f$ has a strong local maximum on $\left(c, y_{2}\right)$ by Proposition 3.8. This is the desired contradiction. The first claim, $f$ is non-increasing on $[a, c]$, follows by a similar argument.

Let us define for $0<a \leq b<\infty$ the annular domains

$$
\begin{aligned}
& A_{n}(a, b):=A(a, b):=\left\{x \in \mathbb{R}^{n}: a<|x|<b\right\} \quad \text { and } \\
& A_{n}[a, b]:=A[a, b]:=\left\{x \in \mathbb{R}^{n}: a \leq|x| \leq b\right\}
\end{aligned}
$$

Lemma 3.10. If $f \in W^{1,1}\left(\mathbb{R}^{n}\right)$ is radial, then $M^{I} f$ does not have a strong local maximum in $\left\{t \in(0, \infty): M^{I} f(t)>|f(t)|\right\}$. 
Proof. Suppose, on the contrary, that $t_{0} \in(0, \infty)$ is a strong local maximum of $M^{I} f$ and $M^{I} f\left(t_{0}\right)>\left|f\left(t_{0}\right)\right|$. Let us choose

$$
\begin{aligned}
& t^{-}:=\sup \left\{t<t_{0}: M^{I} f(t)<M^{I} f\left(t_{0}\right)\right\} \quad \text { and } \\
& t^{+}:=\inf \left\{t>t_{0}: M^{I} f(t)<M^{I} f\left(t_{0}\right)\right\} .
\end{aligned}
$$

By the definition of the strong local maximum, it follows that $t_{0} \in\left[t^{-}, t^{+}\right]$and

$$
M^{I} f(t)=M^{I} f\left(t_{0}\right) \quad \text { for all } t \in\left[t^{-}, t^{+}\right] .
$$

Suppose that $|x|=t_{0}$. Since $M^{I} f\left(t_{0}\right)>\left|f\left(t_{0}\right)\right|$, it follows that there exists a ball $B=B(z, r)$ such that $x \in \bar{B}, r \leq|z| / 4$, and $M^{I} f\left(t_{0}\right)=f_{B}|f|$. Suppose first that $B \not \subset$ $A\left[t^{-}, t^{+}\right]$. In this case, there exists $\varepsilon>0$ such that $\left[t^{-}{ }^{-} \varepsilon, t^{-}\right] \subset\{|y|: y \in \bar{B}\}$ or $\left[t^{+}, t^{+}+\right.$ $\varepsilon] \subset\{|y|: y \in \bar{B}\}$. Especially, it follows by the definition of $M^{I}$ that $M^{I} f(t) \geq f_{B}|f|=$ $M^{I} f\left(t_{0}\right)$ if $t \in\left[t^{-}-\varepsilon, t^{-}\right]$or $t \in\left[t^{+}, t^{+}+\varepsilon\right]$, respectively. Obviously, this contradicts with the choice of $t^{-}$and $t^{+}$. This verifies that $B \subset A\left[t^{-}, t^{+}\right]$. Therefore, it holds by (3.13) that

$$
M^{I} f(y)=M^{I} f\left(t_{0}\right) \quad \text { for all } y \in B .
$$

However, $\left|f\left(t_{0}\right)\right|<M^{I} f\left(t_{0}\right)$ also implies that there exists a ball $B^{\prime}$ with positive radius such that $B^{\prime} \subset B$ and $|f|<M^{I} f\left(t_{0}\right)$ in $B^{\prime}$. Combining this with (3.14) yields the desired contradiction by

$$
\begin{aligned}
M^{I} f\left(t_{0}\right) & =f_{B}|f| \leq \frac{1}{|B|}\left(\int_{B \backslash B^{\prime}}|f|+\int_{B^{\prime}}|f|\right) \\
& <\frac{1}{|B|}\left(\int_{B \backslash B^{\prime}} M^{I} f+\int_{B^{\prime}} M^{I} f\left(t_{0}\right)\right)=M^{I} f\left(t_{0}\right) .
\end{aligned}
$$

The following estimate is well known.

Proposition 3.11. If $f \in W^{1,1}\left(\mathbb{R}^{n}\right)$ is radial and $0<a<b<\infty$, then

$$
\sigma_{n} a^{n-1} \int_{a}^{b}\left|f^{\prime}(t)\right| d t \leq \int_{A(a, b)}|D f(y)| d y \leq \sigma_{n} b^{n-1} \int_{a}^{b}\left|f^{\prime}(t)\right| d t .
$$

\section{The proof of Lemma 3.5}

Let

$$
E:=\left\{x \in \mathbb{R}^{n} \backslash\{0\}: M^{I} f(x)>|f(x)|\right\} \quad \text { and } \quad E_{k}:=E \cap A\left[2^{-k}, 2^{-k+1}\right], \quad k \in \mathbb{N} .
$$

Then $E$ is open, since $M^{I} f$ and $f$ are continuous in $\mathbb{R}^{n} \backslash\{0\}$. A standard argument (see the proof of Proposition 3.2) shows that mapping $M^{I} f$ is locally Lipschitz in 
$E$ and, especially, $D\left(M^{I} f\right)$ exists in $E$. By Lemma 3.1, it suffices to show that $\int_{E}\left|D M^{I} f\right| \leq C_{n}\|D f\|_{1}$.

First, observe that since $|f|$ is radial, it follows that $M^{I} f$ is radial as well. In particular, if

$$
E_{k}^{\mathbb{R}}:=\left\{|x|: x \in E_{k}\right\},
$$

then $x \in E_{k}$ if and only if $|x| \in E_{k}^{\mathbb{R}}$. Since $E_{k}^{\mathbb{R}}$ is open in $\left[2^{-k}, 2^{-k+1}\right]$, we can write

$$
E_{k}^{\mathbb{R}}=\cup_{i=1}^{\infty}\left(a_{i}, b_{i}\right)
$$

such that $a_{i}<b_{i},\left(a_{i}, b_{i}\right)$ are pairwise disjoint and $a_{i}, b_{i} \in \partial E_{k}^{\mathbb{R}}$. In the other words,

$$
E_{k}=\bigcup_{i=1}^{\infty} A\left(a_{i}, b_{i}\right),
$$

and (by the definition of $E_{k}$ ) for all $i \in \mathbb{N}$ it holds that

$$
M^{I} f(x)=|f(x)| \text { if }|x|=a_{i}>2^{-k} \quad \text { and } \quad M^{I} f(x)=|f(x)| \quad \text { if }|x|=b_{i}<2^{-k+1} .
$$

Moreover, since $M^{I} f>|f|$ in $E_{k}$, Lemma 3.10 says that $M^{I} f$ does not have a strong local maximum in $E_{k}^{\mathbb{R}}$. In particular, by Proposition 3.9 there exist $c_{i} \in\left(a_{i}, b_{i}\right)$ such that

$$
\begin{aligned}
\int_{A\left(a_{i}, b_{i}\right)}\left|D M^{I} f(y)\right| d y & \leq \sigma_{n} b_{i}^{n-1} \int_{a_{i}}^{b_{i}}\left|\left(M^{I} f\right)^{\prime}(t)\right| d t \\
& =\sigma_{n} b_{i}^{n-1}\left(M^{I} f\left(a_{i}\right)-M^{I} f\left(c_{i}\right)+M^{I} f\left(b_{i}\right)-M^{I} f\left(c_{i}\right)\right) \\
& \leq \sigma_{n} b_{i}^{n-1}\left(M^{I} f\left(a_{i}\right)-|f|\left(c_{i}\right)+M^{I} f\left(b_{i}\right)-|f|\left(c_{i}\right)\right) .
\end{aligned}
$$

Combining this with (3.15) implies that if $2^{-k}<a_{i}<b_{i}<2^{-k+1}$, then

$$
\begin{aligned}
\int_{A\left(a_{i}, b_{i}\right)}\left|D M^{I} f(y)\right| d y & \leq \sigma_{n} b_{i}^{n-1}\left(|f|\left(a_{i}\right)-|f|\left(c_{i}\right)+|f|\left(b_{i}\right)-|f|\left(c_{i}\right)\right) \\
& \leq \sigma_{n} b_{i}^{n-1} \int_{a_{i}}^{b_{i}}\left|f^{\prime}(t)\right| d t \leq\left(\frac{b_{i}}{a_{i}}\right)^{n-1} \int_{A\left(a_{i}, b_{i}\right)}|D f(y)| d y \\
& \leq 2^{n-1} \int_{A\left(a_{i}, b_{i}\right)}|D f(y)| d y
\end{aligned}
$$

For the case $a_{i}=2^{-k}$ or $b_{i}=2^{-k+1}$, we employ the fact

$$
M^{I} f\left(2^{-k}\right), M^{I} f\left(2^{-k+1}\right) \leq \sup _{y \in A\left(2^{-k-1}, 2^{-k+2}\right)}|f(y)|
$$


to obtain the estimates $\left(a_{i}=2^{-k}\right.$ or $\left.b_{i}=2^{-k+1}\right)$

$$
\begin{aligned}
\int_{A\left(a_{i}, b_{i}\right)}\left|D M^{I} f(y)\right| d y & \leq \sigma_{n} b_{i}^{n-1}\left(M^{I} f\left(a_{i}\right)-|f|\left(c_{i}\right)+M^{I} f\left(b_{i}\right)-|f|\left(c_{i}\right)\right) \\
& \leq \sigma_{n} b_{i}^{n-1} \int_{2^{-k-1}}^{2^{-k+2}}\left|f^{\prime}(t)\right| d t \\
& \leq 2^{3(n-1)} \int_{A\left(2^{-k-1}, 2^{-k+2}\right)}|D f(y)| d y
\end{aligned}
$$

Combining these estimates implies that

$$
\begin{aligned}
& \int_{E_{k}}\left|D M^{I} f(y)\right| d y=\sum_{i=1}^{\infty} \int_{A\left(a_{i}, b_{i}\right)}\left|D M^{I} f(y)\right| d y \\
\leq & 2^{n-1} \sum_{i=1}^{\infty}\left[\int_{A\left(a_{i}, b_{i}\right)}|D f(y)| d y\right]+2\left(2^{3(n-1)}\right) \int_{A\left(2^{\left.-k-1,2^{-k+2}\right)}\right.}|D f(y)| d y \\
\leq & 2^{3 n} \int_{A\left(2^{-k-1}, 2^{-k+2}\right)}|D f(y)| d y .
\end{aligned}
$$

Therefore,

$$
\begin{aligned}
\int_{E}\left|D M^{I} f(y)\right| d y & \leq \sum_{k \in \mathbb{Z}} \int_{E_{k}}\left|D M^{I} f(y)\right| d y \\
& \leq 2^{3 n} \sum_{k \in \mathbb{Z}} \int_{A\left(2^{\left.-k-1,2^{-k+2}\right)}\right.}|D f(y)| d y \\
& =3\left(2^{3 n}\right) \sum_{k \in \mathbb{Z}} \int_{A\left(2^{-k}, 2^{-k+1}\right)}|D f(y)| d y=3\left(2^{3 n}\right)\|D f\|_{1} .
\end{aligned}
$$

This completes the proof.

Then we are ready to prove our main theorem.

Theorem 3.12. If $f \in W^{1,1}\left(\mathbb{R}^{n}\right)$ is radial, then $M f \in W^{1,1}\left(\mathbb{R}^{n}\right)$ and $\|D M f\|_{1} \leq C_{n}\|D f\|_{1}$.

Proof. Let

$$
E:=\left\{x \in \mathbb{R}^{n}: M f(x)>M^{I} f(x), D M f(x) \neq 0\right\} .
$$

It is well known that $M f$ is locally Lipschitz in $\{M f(x)>|f(x)|\}$ (combine e.g. Proposition 3.2 and the fact that $f$ is continuous in $\left.\mathbb{R}^{n} \backslash\{0\}\right)$, implying the existence of $D M f$ in $\{M f(x)>|f(x)|\}$. Since $M f \geq M^{I}$, it holds that $M f(x)=$ 
$\max \left\{M f(x), M^{I} f(x)\right\}$. Therefore, the theorem follows by Lemmas 3.1 and 3.5, if we can show that

$$
\int_{E}|D M f(y)| d y \leq C_{n}\|D f\|_{1}
$$

To show this, observe first that for all $x \in E$ there exist $z_{x} \in \mathbb{R}^{n}$ and $r_{x}>\frac{\left|z_{x}\right|}{4}$ such that $x \in \bar{B}\left(z_{x}, r_{x}\right) \in \mathcal{B}_{x}$. Moreover, since $D M f(x) \neq 0$, Lemma $2.2((2)$ and (3)) says that $x \in \partial B\left(z_{x}, r_{x}\right)$ and $D M f(x) /|D M f(x)|=\left(z_{x}-x\right) /\left|z_{x}-x\right|$. On the other hand, $M f$ is radial and so $D M f(x) /|D M f(x)|= \pm x /|x|$. We conclude that

$$
B_{x}=B\left(c_{x} x,\left|c_{x} x-x\right|\right) \text { for some } c_{x} \in \mathbb{R} .
$$

Firstly, it holds that $c_{x} \geq 0$ for all $x \in E$. To see this, observe that if $c_{x}<0$, then $-x \in$ $B_{x}$ and, since $M f$ is radial, $B_{x} \in \mathcal{B}_{-x}$, implying by Lemma 2.2 that $0=D M f(-x)=$ $D M f(x)$, which contradicts with the assumption $x \in E$. Moreover, $r_{x}=\left|c_{x} x-x\right|=$ $\left|c_{x}-1\right||x|>\left|c_{x} x\right| / 4$ by the assumption, implying that $c_{x}<\frac{4}{5}$ or $c_{x}>\frac{4}{3}$. Summing up, we can write $E=E_{+} \cup E_{-}$, where

$$
E_{+}=\left\{x \in E: c_{x}>4 / 3\right\} \quad \text { and } \quad E_{-}=\left\{x \in E: 0 \leq c_{x}<4 / 5\right\} .
$$

We are going to use different estimates for $D M f(x)$ in $E_{+}$and $E_{-}$. Since $|D M f(x)|=\left|D M f(x) \cdot \frac{x}{|x|}\right|$, it follows from Lemma $2.2(2.8)$ that

$$
|D M f(x)| \leq \frac{1}{|x|} f_{B_{x}}|D| f|(y)||y| d y .
$$

This estimate will be used in $E_{-}$, while in $E_{+}$we will use (easier) estimate $|D M f(x)| \leq f_{B_{x}}|D| f||$ (Lemma 2.2, (1)). We get that

$$
\begin{aligned}
& \int_{E}|D M f(x)| d x \leq \int_{E} \chi_{E_{+}}(x)|D M f(x)|+\chi_{E_{-}}(x)|D M f(x)| d x \\
\leq & \int_{E} \chi_{E_{+}}(x)\left(f_{B_{x}}|D| f|(y)| d y\right)+\chi_{E_{-}}(x)\left(f_{B_{x}}|D| f|(y)| \frac{|y|}{|x|} d y\right) d x \\
= & \int_{E} \int_{\mathbb{R}^{n}} \frac{\chi_{E_{+}}(x) \chi_{B_{x}}(y)|D| f|(y)|}{\left|B_{x}\right|}+\frac{\chi_{E_{-}}(x) \chi_{B_{x}}(y)|D| f|(y)||y|}{\left|B_{x}\right||x|} d y d x \\
= & \int_{\mathbb{R}^{n}}|D| f|(y)|\left(\int_{E_{+}} \frac{\chi_{B_{x}}(y)}{\left|B_{x}\right|} d x+\int_{E_{-}} \frac{\chi_{B_{x}}(y)|y|}{\left|B_{x}\right||x|} d x\right) d y .
\end{aligned}
$$

If $y \in B_{x}$ and $x \in E_{+}$, it follows from the definition of $E_{+}$that $|x| \leq|y|$. Moreover, $y \in B_{x}$ and $x \in E$ imply also that $r_{x} \geq \max \left\{\frac{|y-x|}{2}, \frac{|x|}{3}\right\} \geq \frac{|y|}{5}$. This implies the estimate

$$
\int_{E_{+}} \frac{\chi_{B_{x}}(y)}{\left|B_{x}\right|} d x \leq \int_{B(0,|y|)} \frac{d x}{\omega_{n}(|y| / 5)^{n}} \leq C_{n}, \quad \text { for all } y \in \mathbb{R}^{n} .
$$


On the other hand, if $x \in E_{-}$, then $0 \leq c_{x}<4 / 5$ especially implies that $B_{x} \subset B(0,|x|)$. Therefore, if $x \in E_{-}$and $y \in B_{x}$, then $y \in B(0,|x|)$, and thus $|x| \geq|y|$. Recall also that $r_{x} \geq \frac{|x|}{5}$. Combining these yields that

$$
\int_{E_{-}} \frac{\chi_{B_{x}}(y)|y|}{\left|B_{x}\right||x|} d x \leq|y| \int_{\mathbb{R}^{n} \backslash B(0,|y|)} \frac{d x}{\omega_{n}(|x| / 5)^{n+1}}=C_{n}^{\prime}|y| \int_{|y|}^{\infty} \frac{d t}{t^{2}}=C_{n}^{\prime},
$$

for all $y \in \mathbb{R}^{n}$. This completes the proof.

\section{References}

[AlPe] Aldaz, J.M. and PÉrez LÁzaro, J., Functions of bounded variation, the derivative of the one-dimensional maximal function, and applications to inequalities Trans. Am. Math. Soc., 359 (2007), 2443-2461.

[CaHu] Carneiro, E. and Hughes, K., On the endpoint regularity of discrete maximal operators Math. Res. Lett., 19 (2012), 1245-1262.

[CaMa] E. CARneIRo and J. MADRID. Derivative bounds for fractional maximal operators Trans. Amer. Math. Soc., http://dx.doi.org/10.1090/tran/6844 (electronic).

[HKM] Heinonen, J., Kilpeläinen, T. and Martio, O., Nonlinear potential theory of degenerate elliptic equations Oxford University Press, 1993.

[HM] HajŁasz, P. and MaLY, J., On approximative differentiability of the maximal function, Proc. Am. Math. Soc., 138 (2010), 165-174.

[HO] Hajeasz, P. and Onninen, J., On Boundedness of maximal functions in Sobolev spaces. Ann. Acad. Sci. Fenn., Math., 29 (2004), 167-176.

[Ki] Kinnunen, J., The Hardy-Littlewood maximal function of a Sobolev-function. Isr. J. Math., 100 (1997), 117-124.

[Ku] Kurka, O., On the variation of the Hardy-Littlewood maximal function. Ann. Acad. Sci. Fenn., Math., 40 (2015), 109-133.

[L] Luiro, H., Continuity of the maximal operator in Sobolev spaces. Proc. Am. Math. Soc., 135 (2007), 243-251.

[Ta] TANAKA, H., A remark on the derivative of the one-dimensional Hardy-Littlewood maximal function. Bull. Aust. Math. Soc., 65 (2002), 253-258.

Hannes Luiro

Department of Mathematics and Statistics

University of Jyväskylä

P.O.Box $35(\mathrm{MaD})$

FI-40014 University of Jyväskylä

Finland

hannes.luiro@gmail.com

Received March 1, 2017 